\title{
Hypothèses sur l'auteur. Les enjeux paradoxaux de Robert Pinget
}

\section{Brigitte Ferrato-Combe}

\section{(2) OpenEdition}

12 Journals

Édition électronique

URL : http://journals.openedition.org/recherchestravaux/1295

DOI : 10.4000/recherchestravaux.1295

ISSN : 1969-6434

Éditeur

UGA Éditions/Université Grenoble Alpes

Édition imprimée

Date de publication : 15 mai 2004

Pagination : 157-171

ISBN : 2-9518254-3-9

ISSN : 0151-1874

Référence électronique

Brigitte Ferrato-Combe, « Hypothèses sur l'auteur. Les enjeux paradoxaux de Robert Pinget »,

Recherches \& Travaux [En ligne], 64 | 2004, mis en ligne le 20 mai 2019, consulté le 08 septembre 2020.

URL : http://journals.openedition.org/recherchestravaux/1295; DOI : https://doi.org/10.4000/ recherchestravaux.1295 
Brigitte FERRATO-COMBE

Université Stendhal-Grenoble 3

\section{Hypothèses sur l'auteur Les jeux paradoxaux de Robert Pinget}

Vu le parti pris par l'auteur quel qu'il soit d'en écrire et celui de votre serviteur d'en discourir j'inviterais ceux que ne rebutent ni les aléas de la pensée ni les difficultés du langage à ce qu'il faudrait bien appeler un jeu.

L'Hypothese, p. 2 I.

Entre Fantoine et Agapa, "région enchantée non de France mais de langage $^{\mathrm{I}}$ " où se situent les fictions de Pinget, les personnages d'écrivains abondent. Du premier roman Mabu ou le matériau (1957) au dernier carnet Taches d'encre (1997) en passant par le théâtre, l'œuvre de Pinget est peuplée d' "écrivassiers ", d' "écrivailleurs", de "scribes » en tous genres: Mahu le bafouilleur, Latirail, professeur et romancier réaliste, Mademoiselle Lorpailleur, institutrice qui écrit dans le journal local, Le Fantoniard, Clope éternellement aux prises avec ses dossiers, Monsieur Levert qui rédige interminablement une lettre à son fiston, lettre qu'il n'envoie jamais, et bien d'autres.

Parmi cette population rapidement familière au lecteur, deux personnages se détachent: Mortin, "le seul qui atteigne une dimension mythique ${ }^{2}$ " et Monsieur Songe, dans la dernière partie de l'œuvre ${ }^{3}$. Dans leurs relations

1. Michele Praeger, Les Romans de Robert Pinget, une ecriture des possibles, French Forum Publishers, Lexington, Kentucky, USA, 1987, p. 15.

2. Jean-Claude Liéber, "Mortin l'illustre écrivain", L'Avant-scène Théâtre, I"Is juillet $1987, \mathrm{n}^{\circ} 8 \mathrm{r} 3-814, \mathrm{p} .57$.

3. Il apparaît en 1982 , dans le texte auquel il donne son titre, puis réapparaît dans différents volumes de carnets publiés ultérieurement: Le Harnais, 1984; Charrue, 1985, Du Nerf, 1990 
(inscrites dans la fiction, puisque Mortin est un ami de Monsieur Songe et le premier ou le seul lecteur de ses carnets) et dans la progressive substitution de l'un à l'autre au premier plan de l'œuvre se dessine l'évolution d'une figure de l'auteur qui ne cesse d'être mise en question par Pinget.

Avant d'en analyser les paradoxes, il convient de rappeler les traits principaux de la représentation de l'écrivain, assez concrète et assez contrastée, qui s'élabore au fil de l'œuvre, et qui a été étudiée dans le détail par Michèle Praeger:

La façon dont l'écrivain de la fiction pingétienne est vu par "les autres» correspond à l'envers d'un fantasme, celui de l'écrivain-surhomme, sécrété par l'imaginaire collectif français et dont Barthes a spirituellement rendu compte dans Mythologies [dans le célèbre article "l'écrivain en vacances"] [II] est un paria, un exclu de la société, en partie volontaire [...] S'il est seul, mécompris, méprisé, c'est parce qu'il est véritablement exilé, blessé 4 .

Maniaque, paranoïaque, posant volontiers au "maître", jusqu’à rivaliser avec Faust dans Paralchimie, l'écrivain tel que le représente Pinget est aussi et même le plus souvent - modeste, scrupuleux, anxieux, se contentant de noter les choses vues ou entendues, de rassembler des documents, de rédiger quelques fiches... Obsessionnel toujours, comme le narrateur de Quelqu'un cherchant son papier ou Clope avec son dossier, il entretient une relation constamment problématique avec son œuvre. L'écrivain est un laborieux travailleur attaché à une entreprise ingrate et impossible à achever, tel le personnage central de L'Apocryphe décrit par ses neveux:

Comme disait notre oncle, passer son existence à noircir du papier, suer sang et eau pour rassembler ses idées, numéroter des pages, se relire, récrire, raturer, supprimer et se morfondre enfin sur le résultat n'est pas une grâce, le pauvre homme l'a fait toute sa vie et se demandait sur sa fin quelle mouche l'avait piqué, ils appellent ça la muse, c'est mouise qu'il faudrait dire 5 .

Il est aux prises avec un objet qui lui échappe perpétuellement, qui ne se constitue pas, ou rarement, en œuvre. Toutes ces pages noircies ne font pas un livre. Pierre Taminiaux, après avoir analysé lumineusement ce rapport de l'écrivain fictif à sa production ${ }^{6}$, conclut que «l'écrit, chez Pinget, n'accède jamais au stade de l'impression. Il n'est jamais qu'un manuscrit ${ }^{7}$. Même s'il

et enfin Taches d'encre, 1997, présenté comme "le dernier carnet de monsieur Songen et qui en sera, de fait, l'ultime, puisque Robert Pinget est mort en août 1997, quelques mois après sa parution.

4. Michèle Praeger, Op. cit., p. 29.

5. Robert Pinget, L'Apocryphe, Minuit, 1980, p. 51 .

6. Pierre Taminiaux, Robert Pinget, Seuil, coll. "Les Contemporains", 1994, p. 146.

7. Ibid. 
faut nuancer ce «jamais», le livre sur lequel s'épuise l'écrivain fictif est rarement achevé, rarement publié (lorsqu'il l'est, comme on le verra avec Mortin, l'identité auctoriale n'en est que plus soumise à caution). Le texte n'atteint jamais - même lorsqu'il semblerait pouvoir y prétendre - à la qualité d'œuvre d'art, soumise au regard et au jugement du public, mais demeure dans la sphère privée, au contact intime de l'écrivain. Il est souvent caché, perdu, voire partiellement détruit, il ne circule pas. L'idéal du Livre empêche la réalisation des livres, dans une perspective évidemment mallarméenne. Citant Le Livre à venir de Blanchot, P. Taminiaux met en relief "l'indistinction, ce phénomène associé au texte non marqué par l'empreinte de celui qui l'a écrit. [...] le maître de L'Apocryphe et de L'Ennemi n'est jamais désigné par un nom propre. [...] La création, en ce sens, appartient à tout le monde et à personne: elle implique l'existence d'un objet échappant à toute appropriation ${ }^{8}$.

Est-ce à dire que l'écrivain ne se constitue pas en figure auctoriale, et que Pinget se place dans la perspective d'une disparition de l'auteur? Les analyses précédentes, conduites à la lumière de la «mélancolie blanchotienne» inciteraient à pareilles affirmations. L'étude de quelques pièces de thêatre dans lesquelles apparaît le personnage de Mortin conduit cependant à les nuancer.

A propos de L'Hypothèse, Jean-Claude Liéber écrit: "La pièce traite sur le mode sarcastique le thème pathétique des romans." Sans opposer, ce qui serait très excessif, des romans sérieux à un théâtre ludique, il n'en reste pas moins remarquable que, soucieux d'adopter pour son thêâtre une écriture qui "passe la rampe" et trouvant dans le dialogue theâtral une forme apte à faire apparaître les contradictions, Pinget y représente certains phénomènes avec plus d'alacrité encore que dans ses récits. A le voir convoquer sur scène la quasi-totalité des figures plus ou moins paradoxales de l'auteur, une question se pose: dans quelle mesure le théâtre de Pinget peut-il se lire comme la représentation parodique et "sarcastique " des débats - dont il est contemporain, familier, et dans une certaine mesure partie prenante - sur la disparition, l'effacement et le brouillage de la figure de l'auteur?

Quelques éléments de réponse peuvent être apportés par l'étude de quatre des pièces de théâtre ou dialogues radiophoniques centrés sur le personnage de Mortin: L'Hypothèse (1961), Autour de Mortin (1965), Un Testament bizarre et Mortin pas mort (1986), œuvres choisies pour des raisons à la fois

8. Ibid., p. I5I. 
chronologiques (deux datent du début des années 60 et deux du milieu des années 80) et thématiques: dans ces quatre pièces - qui ont, comme beau-

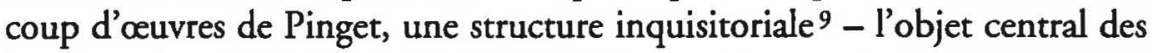
interrogations est Mortin, sa personnalité, ses agissements, les circonstances de sa mort et surtout son existence en tant qu'auteur, son rapport avec un texte, dont la nature et l'origine font l'objet de tous les soupçons.

\section{L'Hypothèse ou la mise en place d'un questionnement}

Dans cette pièce publiée en 196I, créée à la Comédie de Paris en $1964^{\mathrm{IO}}$, Mortin est le personnage unique; mais il se dédouble, se démultiplie: plusieurs images de lui apparaissent en effet projetées sur le mur du fond et prennent même, par moments, la parole.

La didascalie initiale le présente en ces termes:

Une pièce meublée sommairement. A droite un rayonnage servant de bibliothèque. Au fond un petit poêle qui se détache sur un mur très blanc. A gauche une table et un fauteuil. Mortin y est assis, en habit de cérémonie. Très grand, très sec, l'air halluciné. Il essaie de dire par cœeur devant un public supposé un discours dont le texte est placé sous ses yeux. Sur la table également une carafe d'eau et un verre.

Le ton de Mortin est principalement celui de la mémorisation c'est-à-dire ne tenant pas compte des inflexions, pauses et accentuations voulues par la logique de la phrase. Il hésite souvent, se reprend, se réfere au texte. De temps à autre des passages mieux sus qu'il souligne d'effets de voix et de gestes cabotins.

Le débit est assez rapide ${ }^{\mathrm{II}}$.

La dimension parodique et démystificatrice de la pièce est d'emblée manifeste: en situation de discourir devant un public, Mortin, dont le nom rime avec "cabotin", joue un rôle avec emphase. L'objet de son discours est un manuscrit disparu et l'auteur de celui-ci:

9. Elle apparaît très manifestement dans les dialogues qui constituent Autour de Mortin, paru en 1965 , en particulier la partie centrale intitulée Interviews où l'on voit se succéder sinon à la barre du moins au micro huit proches de Mortin censés répondre aux questions d'un interviewer/inquisiteur unique au sujet du défunt. Les contradictions entre les différents témoignages brouillent de plus en plus, au fur et à mesure que les interviews se succèdent, l'image de l'écrivain et notamment l'image de l'auteur. On la retrouve dans les pièces publiées en 1986, Un Testament bizarre et Mortin pas mort, qui rouvrent le dossier Mortin, plus de vingt ans plus tard. Mais elle est également perceptible, quoique de façon plus diffuse, dans les récits et romans comme L'Apocryphe, L'Ennemi ou même Cette voix.

10. Édition de référence: $L$ 'Hypothèse suivi de Abel et Bela, Minuit, 1987.

II. P. II (texte intégral de la didascalie). 
MORTIN. - Dans un sens évidemment on pourrait dire que le manuscrit se trouve au fond d'un puits mais alors pas chez nous puisqu'il n'y aurait plus de puits donc qu'à un moment donné l'auteur se serait déplacé ne fût-ce qu'un temps très court emportant le manuscrit mais pour en faire quoi? D'une part personne ne se souviendrait de l'avoir jamais vu se déplacer d'autre part tous ceux qui l'auraient connu mieux que de vue affirmeraient que son manuscrit était vissé à sa table et qu'il ne pouvait travailler que là ${ }^{12}$.

L'identité de l'auteur est en question dès ces premières phrases; un brouillage s'établit entre distance et proximité, l'auteur étant évoqué à la fois comme un autre et comme très proche. Son identification avec Mortin, d'abord suggérée sur le mode de la dénégation ("mais alors pas chez nous") l'est à nouveau dans la suite de la pièce par la reprise dans le discours de Mortin décrivant l'auteur au travail des termes mêmes de la didascalie évoquant le décor où lui-même se trouve ${ }^{13}$. L'interrogation essentielle formulée par ce début est bien celle du déplacement de l'auteur: le terme est à prendre en un sens à la fois concret (l'auteur sortant de chez lui pour aller jeter son manuscrit dans un puits) et abstrait : s'opère ici un glissement de la figure de l'auteur, qui se dérobe à l'analyse.

Cette interrogation sur l'auteur est intimement liée à celle concernant le destin du manuscrit, qui peut se résumer ainsi : le manuscrit a-t-il été jeté au fond d'un puits? Cette question, triviale en apparence, hante littéralement les quatre pièces étudiées - et au-delà une bonne partie de l'œuvre de Pinget - avec tout un ensemble de questions subsidiaires (où est le puits? quel manuscrit? qui l'a jeté? et bien sûr: pourquoi ?) et peut donc être lue comme l'expression métaphorique (éventuellement parodique) d'une mise en question de la figure auctoriale. La formulation «son manuscrit était vissé à sa table et il ne pouvait travailler que là" suggère une superposition métonymique entre l'auteur et son manuscrit, la métaphore "être vissé à sa table " s'employant en effet habituellement à propos de celui qui fournit un travail intellectuel prolongé et non pas à propos de l'objet ou du résultat de ce labeur. Pinget semble annuler la métaphore en lui substituant l'image du manuscrit effectivement fixé au bois de la table de travail mais il n'y en a pas moins figure, figuration métonymique, et non plus métaphorique, de l'auteur à travers le manuscrit. Cette superposition métonymique est confir-

12. P.13 ( $\mathrm{I}^{\mathrm{r}}$ réplique).

13. "L'auteur donc à sa table écrivant [...] dans un décor qu'on pourrait imaginer à peu près semblable à celui-ci (Geste circulaire) pour ne pas se perdre dans les détails soit une pièce meublée sommairement à droite un rayonnage servant de bibliothèque au fond un petit poêle qui se détache sur un mur très blanc à gauche une table et un fauteuil où serait assis l'auteur écrivant écrivant écrivant quoi toute la question serait là [...] " (p. 26). 
mée par l'ambivalence du "il" anaphorique ("et qu'il ne pouvait travailler que là») qui, syntaxiquement, devrait être co-référentiel au "manuscrit" mais, sémantiquement, renvoie à l'écrivain. L'identification est formulée plus explicitement encore quelques pages plus loin, lorsqu'il est dit de l'auteur que "sans manuscrit il aurait été pratiquement inexistant" (p. Is) et que sont évoqués comme deux événements symboliquement équivalents la destruction du manuscrit et le suicide de l'auteur: « [l'image] de l'auteur penché sur ce puits et y jetant le manuscrit au lieu de s'y jeter lui-même s'imposerait» (p.16).

Quant au puits, lieu fantasmatique de l'œuvre de Pinget où semblent devoir aboutir tous les écrits, s'il ne se trouve pas "chez nous ", comme le prétend Mortin dès ses premiers mots, il n'en est pas moins dans notre langue, puisque selon le proverbe "la vérité est au fond du puits", expression que Pinget reprend à son compte dans les Entretiens:

La perception immédiate qu'il faut retraduire. Que ce soit encore plus vrai que la vérité. Elle est tellement voilée, tellement cachée. La vérité toute nue... elle n'est jamais sortie du puits... pour moi elle est couverte de haillons. (p. 225)

"métaphore insistante de l'enfermement" selon P. Taminiaux (p.155), le puits est à la fois lieu de destruction de l'œuvre, lieu de la mort de l'œuvre et peut-être de l'auteur (dans Autour de Mortin, ce puits est d'ailleurs localisé près du cimetière) mais il est peut-être aussi le seul lieu où l'œuvre peut rester elle-même, au contact de la vérité, sans risquer d'être dénaturée par le regard ou le jugement d'autrui.

L'auteur s'identifie à son manuscrit et disparaît avec lui, ou reste à jamais irrévélé dans l'obscurité du puits: cela serait assez clair, mais dans la pièce de Pinget il en va moins simplement. A l'hypothèse d'un déplacement de l'auteur fait suite aussitôt celle d'un dédoublement du manuscrit entre un original que l'on pourrait déplacer et jeter dans le puits et une copie vissée sur la table de travail: «manuscrit vissé réplique du premier, travail préalable sur ce dernier, recopiage sur le vissé" (p. I8). Duplication du manuscrit à quoi correspond logiquement le dédoublement de l'auteur entre un "auteur effectif" et un "auteur présumé», la multiplication aboutissant à une éventuelle annulation: "d'où nature doublement hypothétique des auteurs ils n'existeraient pas» (p. 23) ${ }^{14}$. Les doutes sur l'existence même de l'auteur et sa pré-

14. "Il y aurait l'auteur effectif ou celui qui de la circonstance du puits aurait fait une hypothèse l'auteur présumé étant celui qui ne fût-ce qu'un temps très court se serait déplacé avec le manuscrit et l'aurait jeté dans le puits... (se réfêre, etc.) lequel auteur ne pourrait être dit relaté par l'effectif car comment celui-ci se saurait-il l'être posant justement l'hypothèse et 
sence sont ouvertement proclamés par Mortin: "l'auteur à sa table [...] n'étant lui-même qu'une hypothèse. (Un temps. Il se lève. Mains appuyées à la table.) L'auteur l'auteur où se trouve l'auteur» (p. 19).

Ces interrogations sur le mode comique trouvent à la fois un écho textuel dans l'emploi du conditionnel, qui place l'ensemble des affirmations concernant l'auteur dans une perspective virtuelle, et un écho visuel dans la projection sur le mur blanc du fond de scène d'une, puis de plusieurs images de Mortin, sans cesse agrandies - images dédoublées, images virtuelles, hypothétiques et cependant bien présentes. Mortin tente d'abord de les éliminer: vers le milieu de la pièce, il sort un revolver, "vise sur l'image et tire. Forte détonation. L'image disparaît." (p.25). Mais c'est finalement lui qui est contraint par leur présence à la destruction de son propre manuscrit (qu'il jette, non dans le puits mais dans le poêle) et à une mise à nu symbolique qui s'apparente à une marche vers la mort sur un mode tragi-comique:

Il continuerait vers le puits qui serait... plus que jamais dans son cœur nécessaire... à sa vie ratée... (Un temps) Tout en marchant il se dirait... il se réciterait... un discours... un discours sur les débris... les débris... où d'autres solitaires comme lui... auraient essayé toute leur vie... de donner un sens à la mort [...] un discours pompier qu'il comprendrait que ce serait celui du manuscrit sous son bras... (Un temps) Et il serait bien étonné... il ne se serait jamais douté... d'avoir fabriqué des symboles... (Un temps) Il trouverait dans les mots bêtes de la dernière heure... la clé des rêves compliqués... qu'il aurait voulu faire briller dans son livre... (Un temps) Il ne regretterait plus rien... plus rien... puisqu'il mettrait... enfin... le doigt sur sa vraie misère... (Un temps) Il verrait défiler dans sa tête... dans sa tête... toutes les occasions perdues de se taire... (Un temps) Elles lui apparaitraient trop tard comme les seules... qui auraient eu une chance d'éclaircir le mystère... le mystère... (Un temps) Les occasions perdues de se taire... (Un temps) Les occasions perdues de se taire... (Un temps) Les occasions perdues... RIDEAU (p. 45-46 FIN)

La représentation de l'échec et de la mort de l'auteur glisse du pathos à la dérision par la prise de conscience de la grandiloquence ("discours pompier ", "symboles") et le recours à un silence dépourvu d'arrière-plan métaphysique ("les occasions perdues de se taire...").

Se joue ainsi une première mort, hypothétique, de l'auteur Mortin, dont les textes suivants vont rejouer la disparition avant de mettre en scène sa résurrection. Avant d'aborder les prochains épisodes, quelques mots s'impo-

présupposant par là le manuscrit, d'autre part des faits consignés dans ledit comment l'auteur effectif pourrait-il être averti sans le fait du présumé le manuscrit allant à vau-l'eau dans le puits donc excluant non seulement la connaissance desdits mais celle même de leur scripteur, et qui parlerait du manuscrit il ne serait au fond du puits que par suite d'une hypothèse d'où nature doublement hypothétique des auteurs ils n'existeraient pas (p. 23). 
sent sur ce nom de Mortin, nom programmatique, qui inclut comme possible la mort du personnage, ainsi que l'écrit Jean-Claude Liéber: "Constat mélancolique. L'écriture est une anticipation de la mort, comme l'indique le nom de Mort-in. L'homme de lettres perd sa singularité au profit d'un universel imaginaire ${ }^{15}$ ». La mélancolie du nom est peut-être cependant à nuancer par d'autres échos, notamment ceux des Scapin, Frontin et autres valets de comédie, genre auquel appartient $L^{\prime} H_{y p o t h e ̀ s e}{ }^{16}$. Par ailleurs, si le nom Mortin laisse présager la mort de l'auteur, il pourrait annoncer également, dans l'onomastique secrète de Pinget, la destruction du manuscrit. C'est ce que révèle l'étude des brouillons d'Autour de Mortin menée par Jean Verrier:

Le début de "Brouillons texte radiophonique" est le début du manuscrit d'un roman inédit de Robert Pinget.

Il s'agit d'un tapuscrit de 234 pages, corrigé à la main, avec quelques pages manuscrites additionnelles intercalées, placé dans une chemise bleue sur la tranche de laquelle est écrit en capitales manuscrites: "MORTIN" et sur la couverture, manuscrit, ce double titre: "Manuscrit raté", flèche vers le bas, "Mortin». Robert Pinget a commenté oralement la signification de cette flèche en m'indiquant que pour lui "Mortin" est l'anagramme de "Manuscrit raté » ${ }^{17}$.

Envisager "Mortin" comme l'anagramme de "Manuscrit raté" (sans prendre, bien sûr, anagramme en un sens strict), c'est associer étroitement l'auteur et son manuscrit, comme deux figures également énigmatiques, également problématiques, également menacées par la destruction, dans un rapport étroitement métonymique. C'est inciter à lire tout ce qui dans l'œuvre de Pinget concerne la disparition, la dispersion, la destruction du manuscrit comme autant d'avanies subies par la figure de l'auteur.

\section{Autour de Mortin (1965) : mise en question de la figure auctoriale}

Le prologue, intitulé Chuchotements, met en scène deux personnages qui en observent par le trou de la serrure un troisième, seul dans une chambre et qui se livre à un comportement plus qu'étrange: il consulte un gros dossier, écrit dans un carnet et finalement s'empoisonne ${ }^{\mathrm{I} 8}$. La série d'interviews qui sui-

I5. Jean-Claude Lieber, art. cit., p. 57.

16. Et peut-être aussi une allusion à Jean Martin, dédicataire de L'Hypothèse.

17. Jean-Claude Liéber et Jean Verrier, "Robert Pinget entre brouillon et apocryphe", Genèses du roman contemporain - Incipit et entrée en écriture, textes réunis par Bernhild Boie et Daniel Ferrer, CNRs ed., 1993, p. 151-184, p. 153 .

18. "Étrange prologue de Autour de Mortin qui nous fait assister, par l'intermédiaire de deux guetteurs, aux préparatifs du suicide de l'écrivain ("Chuchotements"). Encore reste-t-il 
vent, centrés autour de Mortin, écrivain défunt, amènent à penser que le prologue racontait le suicide de celui-ci. Mais d'autres versions de sa mort sont proposées par les différentes personnes de son entourage interrogées: pour Passavoine, buveur lui-même, Mortin était un «ivrogne» et il est mort du "cancer des buv... "; il aurait d'abord essayé de se noyer car il avait "le nœud rasténique" et il était "saoul comme une va..." mais Passavoine l'aurait repêché (p. 89-90) et c'est sa maîtresse, Mimi, qui se serait "pilulée"; la petite-nièce, quant à elle, rapporte la version familiale: "Une sorte d'empoisonnement du sang. Ma mère n'a jamais bien su. Ou du moins prétendait ne pas savoir". Le doute ne concerne pas seulement les conditions de la mort de Mortin mais s'étend à l'ensemble de sa personnalité $e^{19}$ : ivrogne, égoïste, mari tyrannique, peut-être même oncle incestueux: la statue du grand homme ne cesse de s'effriter au fil des interviews.

Surtout, le soupçon se porte sur sa fonction même d'auteur: Mortin est censé avoir écrit et publié un livre sur le capitaine Mortier, qu'il aurait rencontré en Afrique, et en avoir entrepris un autre, resté inachevé, sur le fils de Mortier. Or cette belle image d'auteur reconnu se brouille au fil des témoignages, tous contradictoires et qui ne convergent que sur un point: un manuscrit a été jeté dans le puits.

Dans la version de Cyrille ${ }^{20}$, le garçon de café, Mortin n'est autre que Mortier; l'auteur et le personnage sont une même personne; il y a donc brouillage générique (sous la biographie se cache l'autobiographie), et indéniable écart par rapport à la vérité, mais cela ne porte pas atteinte à l'identité

un doute, tant sur la destruction du manuscrit que sur la réalité du cadavre * (Lieber, art. cit., p. 57).

19. "Le studio radiophonique, dans Autour de Mortin, est une véritable chambre d'accusation. Le soupçon plane sur l'auteur dont l'apparente innocence semble masquer les pires turpitudes (ivrognerie, assassinat, inceste...). Sa renommée est usurpée. Mais la faute n'est pas du ressort de la justice. C'est la littérature elle-même qui semble vouée de toute éternité à la fraude, à "l'apocryphe" " (Lieber, art. cit., p. 57-58).

20. "Et bien... il m'a dit un jour que Mortier c'était lui, il avait trouvé ça pour raconter sa jeunesse et il s'amusait bien lorsque des gens comme par exemple Latirail qui écrit dans Le Fantoniard prétendaient avoir connu Mortier en Afrique ou ailleurs. [...] Il avait écrit son livre en revenant donc très jeune et toute sa vie il a essayé d'écrire le suivant sur le fils Mortier sans y réussir, il disait qu'il n'y croyait plus, il a recommencé pendant des années, il se sentait ligoté. (Un temps) Je lui disais pourquoi ne dites-vous pas la vérité ça serait la meilleure façon d'être libre, il disait toujours transposition transposition, la littérature transpose la poésie l'art le symbole je ne sais plus, quel tourment ç'a été, on peut presque dire qu'il en est mort. ( $U n$ temps) Tout ce qu'il voyait il le rapportait à autre chose pour son roman, un coucher de soleil sur le bois du Furet devenait l'aube sur une oasis, la grosse figure du cantonnier celle d'un chef arabe, un mot quelconque au café était autre chose dit par quelqu'un d'autre..." (p. IIgI20). 
auctoriale. Au contraire, Mortin est décrit comme hanté par des questions proprement littéraires: "la transposition", "la poésie", "l'art", "le symbole": c'est trop sans doute pour que l'ironie en soit absente. Mortin est une figure de l'échec, à cause de son livre impossible sur le fils Mortier, son "fils imaginaire", "son enfant raté» (p. I20), mais cet échec est imputé à des difficultés d'ordre artistique. Mortin est présenté comme un artiste en proie aux affres de la création littéraire. Un doute s'insinue cependant, à propos du puits. Le manuscrit jeté serait-il: «Une première... une première comment dit-on... forme, manière de la vie du fils Mortier qu'il avait prêtée à Johann. Celui-ci l'a jeté dans le puits pour se venger quand il a dû déguerpir " (p. 122) ou "une sorte de dossier contenant des documents" dont la disparition aurait rendu impossible l'achèvement du second livre? L'hypothèse d'un chantage exercé sur Mortin, d'un secret entre Mortin et son domestique Johann, lié à l'existence de documents compromettants et peut-être à l'élaboration de l'œuvre, est seulement suggérée par Cyrille.

Elle est plus clairement évoquée par la logeuse de Mortin, qui reprenant le témoignage de Mademoiselle Meyer - et tout en la désignant comme folle suggère que "le domestique devait collaborer aux écrits de Mortin ":

Elle parlait d'un puits non loin du cimetière où elle aurait vu Mortin sur le point de jeter ses papiers, l'autre le retenait de toutes ses forces. (p. Ios)

Elle a peut-être mal interprété les faits. Mais qu'il y ait eu des papiers et qu'il y ait eu puits, nous n'avons pas de raison d'en douter. (p. 107)

Il faut bien alors envisager l'hypothèse selon laquelle Johann aurait été le nègre de Mortin - et parallèlement la prétendue biographie ne serait qu'une fiction. La figure auctoriale se lézarde sérieusement.

Elle est provisoirement restaurée par la petite nièce de Mortin qui ne peut admettre l'idée d'une fiction et rétablit Mortin dans son autorité de biographe. Selon elle, Daniel Mortier a bel et bien existé, ainsi que son fils James, dont elle serait la fille illégitime:

Ma grand-mère elle-même a bien connu Daniel Mortier lors de son séjour en Algérie. Il était effectivement très lié à mon oncle Alexandre alors recrue là-bas et lui a confié avant de mourir ses carnets de route. Mortin s'est fait un devoir d'écrire la vie de son camarade en s'appuyant sur ces documents irréfutables. (p. 132-133)

Je trouve absurde de laisser planer le doute sur l'existence de mon père. La vie du fils Mortier est bien la sienne. Si l'oncle n'a jamais réussi à la terminer c'est en grande partie à cause de ma mère qui s'y opposait. Mortin a été toute sa vie tenaillé entre le désir de publier ce livre et celui de ne pas déplaire à sa nièce qu'il adorait. (p. 133) 
Le manuscrit existe et pourrait être publié sans l'opposition des cousins (p. 134-5) Cette version ne remet pas en question la fonction auctoriale de Mortin, soulignant la contradiction entre l'auteur et l'homme, les impératifs littéraires et les impératifs familiaux.

Cette interprétation est à son tour violemment réfutée par celle de Latirail, le professeur et critique littéraire dans Le Fantoniard, qui confirme les faits, et notamment l'existence de Mortier, mais jette un jour nouveau sur l'affaire: d'après lui, Mortin serait non pas le dépositaire des carnets de Mortier, soucieux d'honorer la mémoire du disparu, mais un imposteur, qui se serait emparé de l'œuvre de son capitaine ${ }^{21}$. Dans le discours de Latirail, Mortin n'est qu'un usurpateur et la vraie figure d'écrivain devient le capitaine Mortier, valorisé à l'extrême selon tous les clichés habituels: "âme noble", "il faisait confiance à Mortin", "Accorder la moindre valeur à ses notes lui paraissait risible...", "Encore une preuve de son génie... cette désinvolture..." (p. I5s).

Non content d'avoir subtilisé les carnets de Mortier, Mortin aurait de surcroît recouru au service d'un nègre: l'hypothèse de la collaboration plus qu'active de Johann est reprise et développée par Latirail, qui affirme que celui-ci, par vengeance, a jeté les carnets de Mortier dans le puits, empêchant ainsi Mortin de poursuivre son ouvre frauduleuse ${ }^{22}$.

2I. "Le livre sur Mortier est en réalité de Mortier lui-même. J'ai été sous les ordres du capitaine Daniel Mortier en Algérie. J'étais son ordonnance. Il est mort à côté de moi, tué d'un coup de poignard. J'étais son confident et j'en sais long sur lui et sur sa soi-disant amitié pour Mortin. (Un temps) Il avait fait sa connaissance à Alger. Mortin était fasciné par les dons littéraires du capitaine qui consignait toutes ses pensées sur ses carnets de route, je les connaissais par cœur. Un chef-d'œuvre. (Un temps) Il a eu la malencontreuse idée, l'idée néfaste de confier ses notes à Mortin qui le suppliait de les lui faire lire. Mortin s'en est prétendu dépositaire à la mort du capitaine, il a fait état de la lettre où Mortier disait les lui confier, sans rien préciser. (Un temps) J'ai tout essayé pour les ravoir, j'ai fait intervenir madame Mortier, mais elle s'en désintéressait. Mortin a publié sous son propre nom les écrits de Mortier, sans même se référer aux carnets dans une préface. Presque tout a été recopié textuellement. Les remaniements de Mortin sont tous faits au détriment du texte». (p. I53-154)

22. "En résumé, monsieur Latirail, vous avez un jugement sévère sur l'œuvre. Vous prétendez que les sources auraient pour le moins dû être indiquées et vous déniez à Alexandre Mortin le talent nécessaire pour les présenter sous forme de biographie.

- Exact. (Un temps) J'irais même plus loin quant aux... capacités de Mortin. Il n'est pas impossible que les notes de Mortier aient été collationnées et transcrites par Johann. (Un temps) Et le second livre, resté à l'état de manuscrit, entièrement rédigé par lui. [...] Le fait qu'il ait détruit les carnets de Mortier après la parution du premier livre n'infirme pas ce que je dis. Il privait Mortin du même coup, en quittant son service, et de ses sources anciennes qui étaient loin d'être épuisées vu l'importance des carnets, et de ses propres lumières si je puis dire. Cet acte typique de vengeance devait porter un coup décisif aux prétentions littéraires de Mortin et en fait, l'a porté. Mortin n'a jamais réussi à faire son second livre. (p.16I) 
À la mise en question de l'authenticité de l'œuvre succède une critique en règle des "remaniements" opérés par Mortin au texte de Mortier "tous faits au détriment du texte" ( (P I54) et de la qualité des passages écrits par Mortin : "Quant au style des raccords n'en parlons pas" (p. 157). Mortin est ainsi totalement disqualifié comme auteur: l'œuvre qui lui est attribuée ne lui appartient pas et la capacité à en produire une lui est déniée.

Surgit en revanche une nouvelle figure d'auteur, Johann, qui selon Latirail ne se serait pas contenté d'être le nègre de Mortin mais aurait produit des textes que le critique fournit à l'enquêteur en affirmant: "Tenez-vous en au fait. Aucune explication ne vous satisferait. Elle donnerait lieu à de nouvelles contestations. La preuve vous l'avez entre les mains" (p. I62-163). Ces textes constituent la dernière partie d'Autour de Mortin, intitulée "Brouillons». Par ailleurs, on ne peut manquer d'interpréter cette déclaration comme une adresse au lecteur: la preuve de l'existence d'un auteur, c'est le texte même, par conséquent le livre que le lecteur tient entre ses mains confirme l'auctorialité de Robert Pinget.

\section{Un Testament bizarre (1986) : tentative de réhabilitation de la figure auctoriale?}

Si au terme de l'enquête conduite dans Autour de Mortin, celui-ci est non seulement mort mais disparu comme figure d'auteur, vingt ans après, dans Un Testament bizarre, la question se repose et semble recevoir une tout autre réponse. Il s'agit à nouveau d'un dialogue, au sujet d'un écrivain défunt, entre deux hommes, un enquêteur désigné par la lettre $A$ et un témoin, ayant connu le disparu, désigné par la lettre B. L'inquisitoire porte sur différentes versions du testament, et surtout sur un certain manuscrit, dont l'authenticité et la valeur littéraire sont problématiques. Comme dans L'Hypothèse, et plus encore, les versions se multiplient: « un certain manuscrit dont ces messieurs... je veux dire les neveux, avaient chacun soit l'original, à ce qu'ils prétendaient, soit une copie, soit un commentaire, soit..." (B p. ro). L'auteur se dédouble également, non plus par projection de son image sur un mur, mais à travers la multiplication des pseudonymes: «il n'a pas simplifié les choses en signant de noms différents les chapitres ou... comment dire, les parties ou divisions de son écrit» (B p. IO).

Comme dans Autour de Mortin, la question de l'authenticité est clairement posée:

le défunt était-il oui ou non l'auteur de ces documents? (A p. ri)

savoir si le ou les fameux manuscrits, nous voulons dire l'œuvre littéraire, sont authentiques, c'est-à-dire de la main et de l'inspiration du défunt, ou non. (A p. II) 
Il s'agit d'un problème d'attribution; A, qui se présente comme "porteparole de spécialistes de la question qui sont unanimes à reconnaître l'importance de cette œuvre" (p.14) s'interroge sur "l'unité» de ce qu'il définit comme des "mémoires" et affirme clairement son ambition : «Et nous voudrions pouvoir attribuer ces mémoires au défunt sans plus nous préoccuper de ses fantaisies testamentaires" (p. I3). Le problème d'attribution ne se pose qu'en raison de la reconnaissance de la "qualité des écrits du défunt" (p.13); la question est de savoir si cela peut constituer une "œuvre", ce qui suppose la garantie d'une identité auctoriale, laquelle semble ne pouvoir être établie qu'en croisant deux sources d'informations: l'une concernant la personne du défunt, l'autre concernant le style de ses écrits:

n'ayant pas connu le défunt ces spécialistes quelque érudits qu'ils soient ne peuvent se
prononcer sur l'unicité de la source, autrement dit savoir si les différents documents
qu'ils ont étudiés sont de la même personne. Le plagiat de nos jours est chose si répan-
due... N'importe quel écrivassier ne peut avoir les qualités requises pour se pénétrer du
génie d'un auteur, sans doute, mais certains faussaires, vous ne l'ignorez pas, acquièrent
une telle habileté qu'ils arrivent à donner le change. La chose s'est produite plusieurs fois,
caractéristique de notre époque de technicité. (A A.I4-Is)

Ainsi, un auteur serait la conjonction d'une personne et d'un style; le style et l'homme, pour que puisse être vérifiée l'affirmation selon laquelle "le style, c'est l'homme». Cette stabilité de la figure auctoriale est mise en question dans la suite du dialogue: les critères qui permettent d'apprécier la "qualité» littéraire d'un texte, que A expose en bafouillant quelque peu, sont énergiquement contestés par $\mathrm{B}$ :

- Quant à leur critère il se fonde sur... comment dire... une certaine façon de l'auteur de traiter le sujet, qui implique... qui implique une certaine forme d'esprit ou de goût... un certain tempérament lequel se manifeste et par la volonté et par la spontanéité, qu'il s'agisse du choix du vocabulaire ou de la syntaxe dont dépend le ton, le fameux ton, bref par la maitrise de la langue, disons par le style qui n'est autre...

- Que l'homme oui, nous le savons. Définition bien simpliste qui a fait son temps et ne nous satisfait plus. Une personnalité d'artiste peut être très diverse et se manifester de mille façons différentes. L'habileté n'est pas l'apanage de vos seuls faussaires mais peut être aussi celui du génie qui s'étant exprimé ici de telle manière et là de telle autre s'autorise et s'amuse à exploiter lui-même chacune de ces manières. Diversité donc mais source unique. (p.15-16)

On aboutit ainsi à la description d'une œuvre qui joue l'hétérogénéité et la complexité, multiplie les manières et les styles, brouille les frontières entre fiction et autobiographie, dont tous les textes cependant "sont de la même main" (p. 23). Une œuvre au fond qui ressemble beaucoup à celle de Pinget. L'identité auctoriale semble restaurée dans sa plénitude: le manuscrit dispersé est miraculeusement reconstitué, une publication est même envisagée. Nulle grandiloquence cependant, nulle mystique de l'œuvre: le dernier mot 
de la pièce désignant les manuscrits enfin retrouvés est nettement dépréciatif: «Nous viderons la cave!/Joli programme! Tenez, n'oubliez pas vos paperasses..."

\section{Mortin pas mort (1986)}

Tout est remis en question dans Mortin pas mort: un jeune journaliste du Fantoniard s'entretient avec Alexandre Mortin lui-même, censé être mort et enterré depuis vingt ans. Il fait référence à deux enquêtes qui ont eu lieu sur l'écrivain, l'interview, dix ans auparavant, de huit témoins (on reconnaît la structure d'Autour de Mortin) et une autre, plus récente, publiée sous le titre d'Un testament bizarre.

Assistons-nous à une résurrection de l'auteur? Pas vraiment. Si Alexandre Mortin est bien vivant, il nie avoir jamais écrit un seul des livres qu'on lui prête, et pas davantage ses mémoires. Tout ce dont il avoue être l'auteur est une mystification concernant sa propre mort, avec la complicité de sa nièce.

$S$ 'agit-il de la disparition définitive de toute instance auctoriale? Pas davantage. Derrière Mortin se cache son ami Mortier:

Je veux dire que toute sa vie mon vieil ami Mortier s'est amusé, avec mon approbation admirative, à composer des pastiches littéraires ayant pour thème l'existence d'un châtelain ombrageux et désabusé auquel nous avions donné mon nom, et voilà. (p. 46)

Une nouvelle figure paradoxale de l'auteur apparât: celle du pasticheur; figure double de surcroît, renvoyant à une pratique de l'écriture en collaboration, car la part de Mortin semble n'avoir été pas aussi réduite qu'il veut le faire croire: "J'imaginais des canevas durant mes insomnies et il les développait à sa façon" (p. 47). Mortier pasticheur est lui aussi mystificateur, et se révèle le véritable instigateur de l'enquête d'un Testament bizarre (p. 48). Au passage, les journalistes sont roulés dans la farine et les critiques littéraires avec eux; au journaliste désarçonné par tant de "fantaisie", Mortin répond: "Vous êtes jeunes, je le sais, vous devez faire face aux extravagances d'un vieillard qui, lui, n'a plus rien à sauvegarder... que de rares occasions de rire » (p. 49). "Fantaisie»: c'est peut-être le maître mot d'une œuvre qui s'est toujours tenue à distance ironique, bien qu'informée et sans malveillance, de la théorie, et semble avoir, dans les textes dramatiques centrés sur Mortin, mis en scène de façon ludique tous les paradoxes de la figure de l'auteur.

Resterait à se demander si Mortier, l'ami de Mortin, a quelque chose à voir avec Monsieur Songe, dont les carnets sont publiés dans ces mêmes années. On a pu voir se profiler dans l'ombre de l'auteur la silhouette de 
l'ami, dont le rôle est décliné diversement : trahison, imposture ou collaboration; l'œuvre chez Pinget naît dans la solitude mais aussi dans le dialogue, le partage, voire le conflit. Que, dans les derniers textes, Monsieur Songe soit au premier plan et non plus son ami Mortin, n'est-ce pas le signe d'un nouveau déplacement de la figure auctoriale, et d'une évolution dans le rapport de l'auteur à son œuvre? Qu'on en juge par l'avant-propos de Monsieur Songe (I982) ${ }^{23}$ :

Pendant une vingtaine d'années je me suis délassé de mon travail en écrivaillant les histoires de monsieur Songe. Les voici réunies et mises au point en un volume qui est, je le répète, un divertissement.

Du «travail" au "divertissement", «joli programme» comme dirait Pinget.

23. Monsieur Songe réapparaît dans différents volumes de carnets publiés ultérieurement: Le Harnats, 1984; Charrue, 1985, Du Nerf, 1990 et enfin Taches d'encre, 1997, dans lequel figure à nouveau un avant-propos :

"Ce petit livre est le dernier carnet de monsieur Songe.

Il s'y montre à découvert, naiff, las des belles formules et peu soucieux de se contredire et de se répéter.

Peut-être aurait-il dû garder ces feuilles par-devers lui mais le besoin de faire part de ses incertitudes a été le plus fort.

Qu'on lui pardonne une dernière fois». 